\title{
Reflex sympathetic dystrophy of the lower extremity in tetraplegia: case report
}

\author{
Todd P Lefkoe and Diana D Cardenas \\ Northwest Regional SCI System, University of Washington, Department of Rehabilitation Medicine, Seattle, \\ Washington, USA
}

\begin{abstract}
Reflex sympathetic dystrophy (RSD) of the upper extremities has been reported to occur following complete and incomplete injuries of the cervical cord. Such reports describe the value of the three-phase radionuclide bone scan (TPBS) in differentiating RSD from pain of other sources. To our knowledge, RSD of the lower extremities has not been reported in a patient with tetraplegia. We report a case of lower extremity RSD in a patient with a complete traumatic injury of the cervical cord. The case illustrates the use of the TPBS to differentiate RSD from heterotopic ossification (HO) in the lower extremities. The successful use of the alpha-adrenergic blocker, phenoxybenzamine, in the treatment of RSD is described. Follow-up to 30 months has shown no evidence of recurrence, and complete resolution of the scintigraphic findings.
\end{abstract}

Keywords: reflex sympathetic dystrophy; lower extremities; tetraplegia; pain syndromes; phenoxybenzamine

\section{Introduction}

Reflex sympathetic dystrophy (RSD) is a term that has been applied to a great variety of seemingly unrelated disorders variously described as causalgia. Sudeck's atrophy, peripheral acute trophoneurosis, post-traumatic osteoporosis, minor causalgia, shoulder-hand syndrome and reflex dystrophy. These syndromes have similar clinical manifestations but may differ in their precipitant. RSD is characterized by pain (usually excessive or prolonged), vasomotor and other autonomic disturbances, soft tissue swelling and trophic changes. ${ }^{1}$ In some patients, pain is mild but vasomotor disturbances are marked. In others, edema is severe while the incidence of vasomotor disturbances and pain vary. Pain and vasomotor phenomena are present without any evidence of swelling in some patients.

Numerous authors have staged the various syndromes and, despite some differences, all suggest three stages. $^{2,3}$ The first stage is characterized by pain, hyperesthesia, edema and tenderness. The second stage is characterized by a gradual decrease in pain, spread of edema, trophic changes of hair, nails and skin and radiographic changes. In the final stage, atrophy predominates while vasomotor problems are absent.

Reports of RSD in the upper extremity after spinal

Correspondence: TP Lefkoe, Orthopaedics and Rehabilitation Health Care Service, Fletcher Allen Health Care, University of Vermont School of Medicine, 1 South Prospect Street, Burlington, VT 05401, USA cord injury describe the value of the three-phase bone scan (TPBS) in differentiating RSD from pain of other sources. ${ }^{4}$ The purpose of this case report is threefold: (1) to report the occurrence of lower extremity RSD in a patient with complete tetraplegia; (2) to describe the use of the TPBS to differentiate RSD from heterotopic ossification (HO) of the lower extremities and (3) to report the successful treatment of lower extremity RSD with the alpha-adrenergic blocker, phenoxybenzamine.

\section{Case report}

A 25 year old male with C6 complete tetraplegia secondary to a diving accident 3 years previously, presented to the emergency room (ER) with complaints of chest pain, shortness of breath and a 2-3 day history of right ankle swelling. The past medical history was significant for right hip HO, first noted 4 months after spinal cord injury and resected 2 years later to restore hip range of motion and function.

On examination, there was right ankle edema, and the right calf was warm and measured $2.25 \mathrm{~cm}$ in circumference greater than the unaffected contralateral limb. Diagnostic studies included a venous duplex scan of the right lower extremity, radiographs of the right knee and tibia and arterial blood gas, all of which were unremarkable. The patient was started on indomethacin $25 \mathrm{mg}$ po TID and was discharged from the ER. 
The next week, the patient was seen in the Rehabilitation Medicine Clinic. His right calf remained $3 \mathrm{~cm}$ in circumference greater than the left, with continued edema of his right foot and ankle. The patient was carefully questioned about his recollection of local trauma to the area. He was able to recall twisting the right ankle during a transfer, approximately one day prior to the onset of swelling. X-rays of the right foot and ankle demonstrated diffuse osteopenia and soft tissue swelling. A Technetium$99 \mathrm{~m}$ bone scan was completed, revealing a diffusely increased uptake distal to the right knee with juxtaarticular accentuation in the foot and ankle on delayed imaging of the limb, consistent with reflex sympathetic dystrophy (Figures 1 and 2). Activity about the right hip, from which heterotopic ossification had been resected 10 months before, was markedly reduced in comparison to preoperative scans. Laboratory values drawn at the time of the clinic visit demonstrated an alkaline phosphatase level of 60 (normal 35-100 U/L) and erythrocyte sedimentation rate of $1 \mathrm{~mm} / \mathrm{h}$.

The indomethacin was stopped and the patient was started on phenoxybenzamine at a dose of $10 \mathrm{mg}$ po qd for 4 days, then $10 \mathrm{mg}$ po bid. He reported prompt resolution of edema and lower extremity pain within the next few days. At 3 months follow-up, the calf measurements were equal and the patient remained pain-free. The phenoxybenzamine was then tapered off over 2 weeks. No further symptomatic recurrences ensued. A repeat bone scan at 30 months demonstrated resolution of the scintigraphic findings in the distal right lower extremity (Figure 3).

\section{Discussion}

Within the last 10 years, several authors have contributed to the increasing recognition of RSD following cervical spinal cord injury. Wainapel described the development of upper extremity RSD in two patients with a clinical presentation of central cord

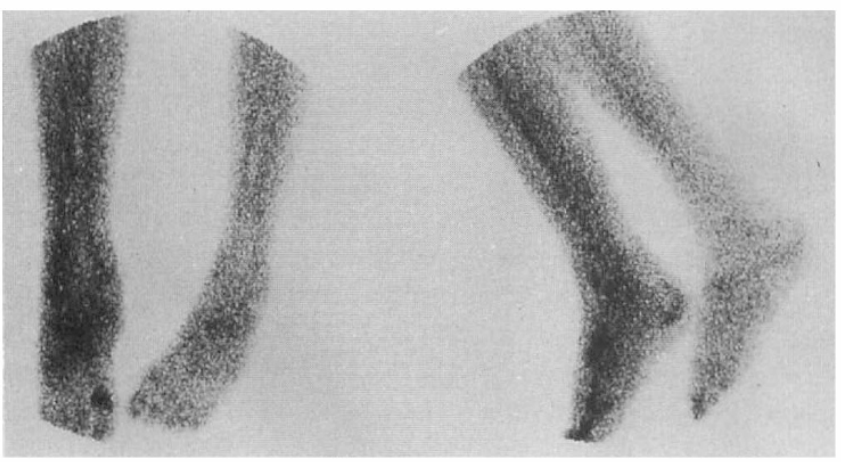

Figure 1 Abnormal TPBS, delayed phase, anterior view demonstrating diffusely increased uptake in the right lower extremity syndrome following traumatic cervical myelopathy. ${ }^{5}$ Gellman and colleagues identified a $10 \%$ incidence of RSD in their series of 60 patients with tetraplegia. ${ }^{4}$ In each case, RSD was noted only in the upper extremities. Additional studies detail the occurrence of upper extremity RSD in small series of patients with

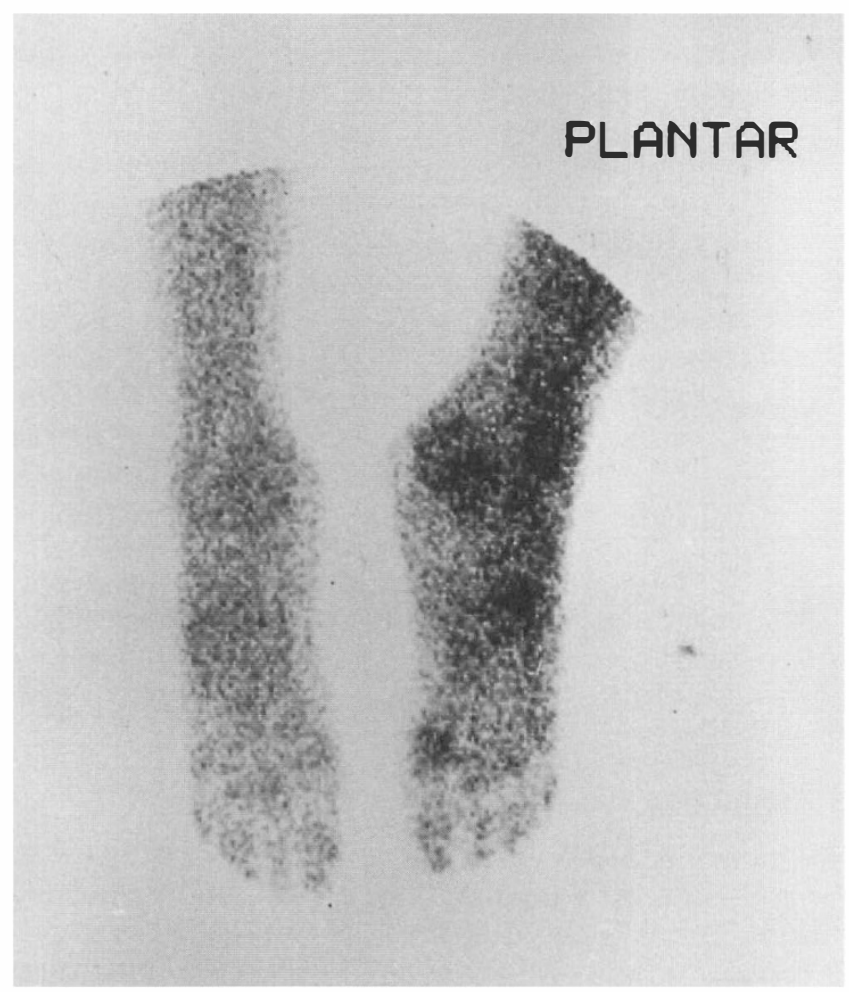

Figure 2 Abnormal TPBS, delayed phase, plantar view demonstrating diffuse juxta-articular uptake pattern in the right foot and ankle

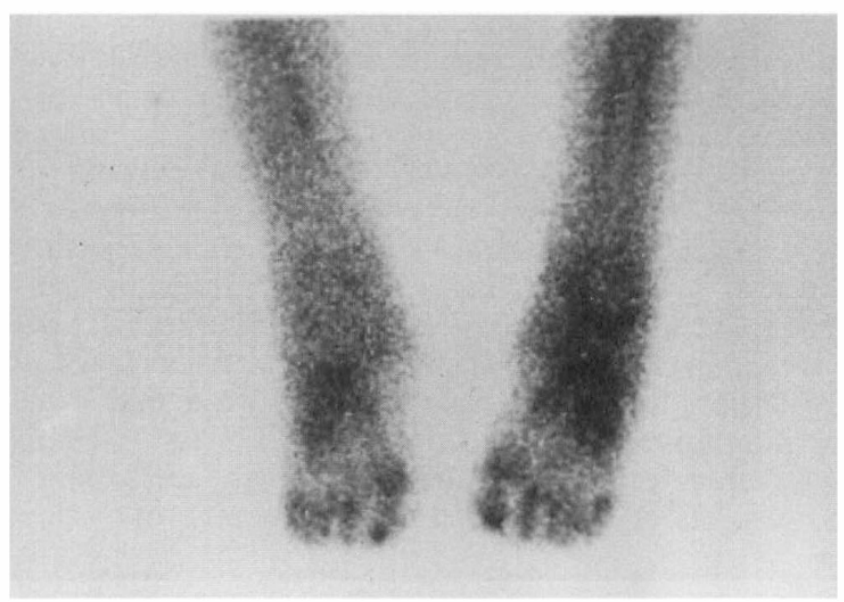

Figure 3 Delayed phase TPBS, anterior view at 30 months, demonstrating normal scintigraphic uptake in the right lower extremity 
both complete and incomplete tetraplegia, on the basis of clinical, radiographic and scintigraphic data. ${ }^{6,7}$ To our knowledge, however, there exists no previous report of RSD involving the lower extremities in patients with tetraplegia.

The diagnostic features of RSD have long been described $^{2,8}$ and standardized criteria for the diagnosis proposed. ${ }^{9}$ That the diagnosis is not made more frequently in patients with tetraplegia may simply reflect an incidence that is lower than that seen for other neurological insults, such as CVA. ${ }^{10}$ Alternatively, the true incidence of RSD may be masked by the natural course and treatment of spinal cord injuries. Early and aggressive mobilization and range of motion have been suggested to be prophylactic against the development of RSD. ${ }^{5}$ It has also been proposed that the use of steroids in the initial post-traumatic period may have a protective effect. ${ }^{5}$ This theory is based on several studies that have shown beneficial treatment effects for systemic steroid use in neurologically intact patients with RSD. ${ }^{3,9}$ The theory fails to account for the fact that, while the routine use of systemic steroids following spinal cord injury has come about only within the last $10-15$ years, reports of RSD in patients with spinal cord injuries have failed to show a requisite decline during this period. It also does not explain the relative absence of reported cases in this population prior to the widespread use of steroids.

Since RSD is a clinical syndrome, it follows that the diagnosis can be established by history and physical examination. However, diagnostic uncertainty often surrounds the presence of pain syndromes in tetraplegia. Pain is a frequent sequela of traumatic spinal cord injury, with an estimated incidence of up to $94 \%$ in some series. ${ }^{11}$ Some have suggested that the reported incidence of RSD in tetraplegia is low because it is misdiagnosed as pain of alternate etiology. ${ }^{5}$ Dysesthetic, or central, pain can be easily confused with RSD, and it appears that etiology, level and completeness of injury cannot be used prognostically to differentiate between the two. ${ }^{11}$ Autonomic dysfunction resulting from cervical cord injury may also add to the diagnostic confusion in the patient with suspected RSD.

The differential diagnosis in this case report included deep venous thrombosis (DVT) and HO both common problems following spinal cord injury. Recent investigations have demonstrated that these two clinical entities may occur concurrently following spinal cord injury, ${ }^{12}$ while others have shown a significant association between the occurrence of DVT and HO following traumatic spinal cord injury. ${ }^{13}$ That the inflammatory process associated with HO can mimic the clinical presentation of DVT further clouds the issue. ${ }^{14}$ Heterotopic ossification is estimated to occur in $20-40 \%$ of patients with spinal cord injury. ${ }^{15}$ The ankle is rarely affected, however. ${ }^{16}$ Only by careful questioning was the history of ankle trauma elicited. The importance of this clinical point cannot be overstated, as trauma to the insensate limb can easily be overlooked by both patient and physician. Therefore, although RSD appears to be a relatively uncommon clinical entity, it must be considered in the differential diagnosis of unilateral lower extremity swelling in the patient with tetraplegia.

Given the overlap in clinical presentation and the frequent ambiguity of clinical findings, practitioners have turned increasingly toward the use of the threephase bone scan to support and confirm the diagnosis of RSD. Early accounts of the diagnostic use of bone scintigraphy in RSD reported a sensitivity of $60 \%$ and a specificity of $86 \% .{ }^{17}$ Subsequent studies have proposed more strict criteria for the clinical diagnosis and interpretation of diagnostic scans. A diffuse increase in tracer activity with generalized juxtaarticular uptake on delayed phase scans has been shown to be highly sensitive and specific for RSD of the foot ${ }^{18}$ and hand. ${ }^{19-21}$ Application of these criteria to clinical studies of patients with RSD have yielded sensitivities of $96-100 \%^{18-21}$ and specificities of $80 \%-98 \%{ }^{18-21}$ for delayed phase scans. None of these studies, however, contained patients with spinal cord injuries. Upper extremity RSD following spinal cord injury is thought to be infrequent and is presumably quite rare in the lower extremity.

The treatment of RSD is the source of continuing debate. In this case, the patient was successfully treated with oral phenoxybenzamine, a postsynaptic alpha $_{1}$-blocker and presynaptic alpha ${ }_{2}$-blocker. Ghostine et al reported the use of this agent in 40 consecutive cases of causalgia resulting from missile or shrapnel wounds, including five patients with cauda equina injury. ${ }^{22}$ Phenoxybenzamine was started within 2 weeks after the onset of causalgia in $80 \%$ of patients in the series. Total resolution of causalgic pain was achieved within 18 days of treatment in each case. No recurrence of causalgia was seen in the series at up to 6 years follow-up. Medication side effects included orthostatic hypotension, with subjective complaints of light-headedness in $43 \%$ of cases. ${ }^{22}$ In roughly half of these patients, the hypotension was persistent and required treatment with stockinettes or an abdominal binder for the duration of the phenoxybenzamine course.

The average length of treatment reported by Ghostine was 6-8 weeks, although some patients received the drug for up to 3 months. ${ }^{22}$ The starting dose of phenoxybenzamine was $10 \mathrm{mg}$ po tid, increasing by increments of $10 \mathrm{mg}$ qd every 2 days until either complete resolution of pain or postural hypotension were seen. The average daily dose needed for pain relief was $80 \mathrm{mg}$. In this case report, treatment was initiated with a smaller dose $(10 \mathrm{mg}$ po qd) to avoid hypotensive side effects in a patient with known autonomic dysfunction secondary to spinal cord injury. After 4 days, the dose was increased to $10 \mathrm{mg}$ po bid. This resulted in complete pain relief and resolution of edema without orthostasis. Treatment was continued for 3 months, at which time the phenoxybenzamine was slowly tapered. 
Phenoxybenzamine has previously been shown to be effective in the treatment of Sudeck's atrophy. ${ }^{23}$ The rationale for the authors' use of this agent in the case report is largely based on the reported success of chemical $^{24}$ and surgical sympathectomy ${ }^{25,26}$ in the treatment of RSD. That the postganglionic sympathetic nervous system is actively involved in the pathogenesis of at least some forms of RSD seems clear. However, the mechanisms underlying the generation and maintenance of these pain syndromes are still unclear. To date, no reproducible, doublebinded controlled studies yet exist to support the efficacy of any single agent or surgical procedure in treating RSD.

\section{Conclusions}

This is the first reported case of RSD occurring in the lower extremity of a patient with tetraplegia. The diagnosis of RSD in the patient with spinal cord injury represents an unique challenge, given the potential for diagnostic confusion and dysesthetic pain and more common complications such as HO or DVT. A recent history of trauma to an insensate limb suggests the diagnosis. The delayed image of the three-phase bone scan is both sensitive and specific for RSD and can be used to support or confirm the clinical diagnosis. This report demonstrates the successful use of the alphablocker, phenoxybenzamine, to treat RSD.

\section{References}

1 Dotson RM. Causalgia-reflex sympathetic dystrophy-sympathetically maintained pain: myth and reality. Muscle and Nerve 1993; 16: $1049-1055$.

2 Steinbrocker O, Spitzer N, Friedman HH. The shoulder-hand syndrome in reflex dystrophy of the upper extremity. Ann Intern Med 1947; 92: 22-49.

3 Kozin F, McCarty DJ, Sims J, Genant H. The reflex sympathetic dystrophy syndrome. I. Clinical and histologic studies: evidence for bilaterality, response to corticosteroids and articular involvement. Am J Med 1976; 60: 321-331.

4 Gellman $\mathrm{H}$ et al. Reflex sympathetic dystrophy in cervical spinal cord injury patients. Clin Orthop 1988; 233: 126-131.

5 Wainapel SF. Reflex sympathetic dystrophy following traumatic myelopathy. Pain 1984; 18: $345-349$.

6 Cremer SA, Maynard F, Davidoff G. The reflex sympathetic dystrophy syndrome associated with traumatic myelopathy: report of 5 cases. Pain 1989; 37: 187- 192.
7 Philip PA, Philip M, Monga TN. Reflex sympathetic dystrophy in central cord syndrome: case report and review of the literature. Paraplegia 1990; 28: $48-54$.

8 Miller DS, DeTakats G. Post-traumatic dystrophy of the extremities-sudeck's atrophy. Surg Gynec and Obst 1941; 75: $558-581$.

9 Kozin F et al. The reflex sympathetic dystrophy syndrome III. Scintigraphic studies, further evidence for the therapeutic efficacy of systemic corticosteroids, and proposed diagnostic criteria. Am J Med 1981; 70: 23-30.

10 Tepperman PS et al. Reflex sympathetic dystrophy in hemiplegia. Arch Phys Med Rehabil 1984; 65: 442- 447.

11 Davidoff $\mathrm{G}$ et al. Function-limiting dysesthetic pain syndrome among traumatic spinal cord injury patients: a cross-sectional study. Pain 1987; 29: 39-48.

12 Bradleigh LH et al. Deep venous thrombosis associated with heterotopic ossification. Arch Phys Med Rehabil 1992; 73: 293 294.

13 Colachis SC, Clinchot DM. The association between deep venous thrombosis and heterotopic ossification in patients with acute traumatic spinal cord injury. Paraplegia 1993; 31: 507-512.

14 Orzel JA, Rudd TG, Nelp WB. Heterotopic bone formation (myositis ossificans) and lower-extremity swelling mimicking deep-venous disease. J Nucl Med 1984; 25: 1105-1107.

15 Lynch C, Pont A, Weingarden SI. Heterotopic ossification in the hand of a patient with spinal cord injury. Arch Phys Med Rehabil 1981; 62: $291-293$.

16 Hernandez AM et al. The para-articular ossifications in our paraplegics and tetraplegics: a survey of 704 patients. Paraplegia 1978; 16: $272-275$.

17 Kozin F et al. Bone scintigraphy in reflex sympathetic dystrophy syndrome. Radiology 1981; 138: 437-443.

18 Holder LE, Cole LA, Myerson MS. Reflex sympathetic dystrophy in the foot: clinical and scintigraphic criteria. Radiology 1992; 184: 531 - 535 .

19 Holder LE, Mackinnon SE. Reflex sympathetic dystrophy in the hands: clinical and scintigraphic criteria. Radiology 1984; 152: $517-522$.

20 Mackinnon SE, Holder LE. The use of three-phase radionuclide bone scanning in the diagnosis of reflex sympathetic dystrophy. $J$ Hand Surg 1984; 9A: 556- 563 .

21 Kline SC, Holder LE. Segmental reflex sympathetic dystrophy: clinical and scintigraphic criteria. J. Hand Surg 1993; 18: $853-$ 859.

22 Ghostine SY et al. Phenoxybenzamine in the treatment of causalgia-report of 40 cases. J Neurosurg 1984; 60: $1263-1268$.

23 Lankford LL. Reflex Sympathetic Dystrophy. In: Omer GE Jr, Spinner M (eds). Management of Peripheral Nerve Problems. WB Saunders: Philadelphia 1980, pp 216-244.

24 Hoffman $\mathrm{J}$ et al. Effect of sympathetic block demonstrated by triple-phase bone scan. J Hand Surg 1993; 18A: 860-864.

25 AbuRahma AF et al. Sympathectomy for reflex sympathetic dystrophy: factors affecting outcome. Ann Vasc Surg 1994; 8: $372-379$.

26 Herz DA et al. Second thoracic sympathetic ganglionectomy in sympathetically maintained pain. J Pain Sympton Manage 1993; 8: $483-491$. 